\title{
Limits in the use of cPTIO as nitric oxide scavenger and EPR probe in plant cells and seedlings
}

\section{Stefano D'Alessandro ${ }^{1}$, Bianca Posocco ${ }^{2}$,Alex Costa ${ }^{1,3}$, Georgia Zahariou ${ }^{2}$, Fiorella Lo Schiavo ${ }^{1}$, Donatella Carbonera $^{2}{ }^{*}$ and Michela Zottini ${ }^{1}$ *}

${ }^{1}$ Department of Biology, University of Padova, Padova, Italy

${ }^{2}$ Department of Chemical Sciences, University of Padova, Padova, Italy

${ }^{3}$ Department of Biosciences, University of Milan, Milano, Italy

\section{Edited by:}

John Hancock, University of the West of England, UK

\section{Reviewed by:}

Vasileios Fotopoulos, Cyprus University of Technology, Cyprus Renaud Brouquisse, Institut National de la Recherche Agronomique, France

\section{${ }^{*}$ Correspondence:}

Michela Zottini, Department of

Biology, University of Padova, Via Ugo Bassi 58/B, 35131 Padova, Italy e-mail: michela.zottini@unipd.it; Donatella Carbonera, Department of Chemical Sciences, University of Padova, Via Marzolo 1, 35131

Padova, Italy

e-mail: donatella.carbonera@unipd.it
Over the last decade the importance of nitric oxide (NO) in plant signaling has emerged. Despite its recognized biological role, the sensitivity and effectiveness of the methods used for measuring NO concentration in plants are still under discussion. Among these, electron paramagnetic resonance (EPR) is a well-accepted technique to detect NO. In the present work we report the constraints of using 2-4-carboxyphenyl-4,4,5,5-tetramethylimidazoline1-oxyl-3-oxide (CPTIO) in biological samples as spin trap for quantitative measurement of NO. EPR analyses on Arabidopsis cell cultures and seedlings show that CPTIO(NNO) is degraded in a matter of few minutes while the (INO) compound, produced by CPTIO and $\mathrm{NO}$ reaction, has not been detected. Limitations of using this spin trap in plant systems for quantitative measurements of $\mathrm{NO}$ are discussed. As NO scavenger, CPTIO is widely used in combination with 4-amino-5-methylamino-2', $7^{\prime}$-difluorofluorescein (DAF-FM) fluorescent dye in plant research. However, the dependence of DAF-FM fluorescence on CPTIO and NO concentrations is not clearly defined so that the range of concentrations should be tightly selected. In this context, a systematic study on CPTIO NO scavenging properties has been performed, as it was still lacking for plant system applications. The results of this systematic analysis are discussed in terms of reliability of the use of cPTIO in the quantitative determination and scavenging of $\mathrm{NO}$ in plants and plant cultured cells.

Keywords: plant, nitric oxide, cPTIO, electron paramagnetic resonance (EPR), NO scavenger, DAF-FM, Arabidopsis

\section{INTRODUCTION}

Nitric oxide (NO) is a signal molecule involved in controlling both physiological processes and stress responses (Mur et al., 2013). It plays an important role in root organogenesis and development (Correa-Aragunde et al., 2004) and in auxin signaling (Kramer and Bennett, 2006) and perception (Terrile et al., 2012). In response to pathogen attacks, NO turns to be a key molecule in the hypersensitive response (HR) and programmed cell death (PCD) events (Wang et al., 2013). Recently, the role of $\mathrm{NO}$ has also been investigated in abscisic acid (ABA)-associated response of guard cells to pathogens (Ye et al., 2013).

The central role of NO in plants is corroborated by the presence of many different enzymatic and non-enzymatic sources (Gupta et al., 2010). However, the controversial existence of NO synthaselike enzymes makes it difficult to define the specific NO source engaged in a specific physiological process and to understand how it is involved in it. For this reason, in order to establish whether and where NO is produced by specific cells and tissues, plant researchers rely on several indirect methods of analysis. Many of the methods developed for NO detection capitalize on its high diffusibility as well as on its broad spectrum of chemical reactivity. However, in biological systems, the use of these methods is limited by the short half-life of the molecule (Woldman et al., 1994; Gupta and Igamberdiev, 2013).
Electron paramagnetic resonance (EPR) is a well-accepted spectroscopic technique to detect $\mathrm{NO}$ in a liquid phase (Hogg, 2010). This technique is selective for monitoring radical species. In principle, being $\mathrm{NO}$ a radical, a direct measurement by EPR should be possible; however, due to its fast spin relaxation time, it cannot be detected. Therefore, the methods of NO detection in solution through EPR are based on the trapping of NO with the formation of stable paramagnetic species (Hogg, 2010). As a matter of fact, in biological samples spin trapping methods are largely used for detection of short-living radicals such as $\mathrm{O}_{2}^{-}, \mathrm{OH}^{\bullet}$, both in vivo and in vitro (Berliner, 2000). Spin trapping is necessary since conventional EPR requires a steady state concentration of the free radical higher than $0.01 \mu \mathrm{M}$.

Iron dithiocarbamates have been widely used as spin traps, due to their high affinity for NO. The formation of stable nitrosyl iron-dithiocarbamate complexes gives a three-line EPR spectrum at room temperature, characterized by the hyperfine interaction with the $\mathrm{N}$ nucleus of NO (Vanin et al., 2000). However, the use of iron dithiocarbamates is problematic for quantitative NO determination, either in planta or in cultured cells, due to the interference of nitrites and nitrates that can produce $\mathrm{NO}$ under the reducing experimental conditions required for this assay (Hogg, 2010).

Alternatively, nitroxide spin traps have been tested in vitro and in animal cell systems (Haseloff etal., 1997). A well-known nitroxide spin trap for $\mathrm{NO}$ used in biological 
samples is 2-4-carboxyphenyl-4,4,5,5-tetramethylimidazoline-1oxyl-3-oxide (cPTIO) that belongs to the nitronyl nitroxides (NNO) compounds. NNOs are stable organic radicals that react with $\mathrm{NO}$, with rate constant of about $10^{4} \mathrm{M}^{-1} \mathrm{~s}^{-1}$, forming imino nitroxides (INO) with a significant change in the associated EPR spectra (Yoshioka et al., 1996). In fact, following this reaction, the number of lines in the EPR spectra changes from five to seven.

For its chemical properties cPTIO has been commonly used also as a NO scavenger in combination with 4-amino-5-methylamino$2^{\prime}, 7^{\prime}$-difluorofluorescein (DAF-FM) fluorescent dye, although many pitfalls have been evidenced (Vitecek et al., 2008; Rumer et al., 2012). Conversion of DAF-FM to the corresponding triazole forms (DAF-FM-T) by reaction with NO causes little changes in the absorbance maxima but greatly increases the fluorescence quantum efficiency. DAF-FM dyes react with $\mathrm{N}_{2} \mathrm{O}_{3}$, a by-product of $\mathrm{NO}$ oxidation, with a resulting increase in fluorescence, dependent on NO concentration. cPTIO is used as a scavenger of NO, to remove the increase of DAF-FM fluorescence, and prove in this way the production of $\mathrm{NO}$ in the system. However, it has also been shown that cPTIO, under particular experimental conditions, may facilitate formation of $\mathrm{N}_{2} \mathrm{O}_{3}$ by increasing the rate of $\mathrm{NO}$ oxidation, thus inducing an increase, instead of a decrease, of DAF-FM fluorescence (Arita et al., 2006). In fact, cPTIO oxidizes NO forming $\bullet \mathrm{NO}_{2}$ radical $\left(\mathrm{NO}+\mathrm{cPTIO} \rightarrow \bullet \mathrm{NO}_{2}+\mathrm{cPTI}\right)$, which in turn can react with $\mathrm{NO}$ to form $\mathrm{N}_{2} \mathrm{O}_{3}\left(\mathrm{NO}_{2}+\mathrm{NO} \rightarrow \mathrm{N}_{2} \mathrm{O}_{3}\right)$. The sensitivity of the fluorescence intensity to $\mathrm{pH}$ and ascorbic acid was also considered as a source of uncertainty in the detection of $\mathrm{NO}$ in plants.

Despite these intrinsic problems, the advantages of cPTIO to be specific for NO and cell permeable (Vitecek et al., 2008), along with its widespread use in plant experiments, prompted us to perform a systematic study on cPTIO NO scavenging properties, since a detailed analysis was still lacking regarding applications to plant systems.

\section{RESULTS}

\section{cPTIO AS A SPIN TRAP FOR NO DETECTION IN PLANTS}

We have evaluated the use of cPTIO as NO spin trap in plants by analyzing its EPR spectrum in different experimental conditions. In Figure 1, the reference spectrum of $100 \mu \mathrm{M}$ cPTIO(NNO) in water is shown. Based on the stoichiometry of the reaction between cPTIO and NO (1:2) (Hogg etal., 1995) and on the NO release stoichiometry by the NO donor ((Z)-1-(N-Methyl- $N$-[6-( $N$-methylammoniohexyl)amino])-diazen-1-ium-1,2-diolate) MAHMA NONOate, $200 \mu \mathrm{M}$ of the NO donor was used to obtain a saturated signal corresponding to about $100 \mu \mathrm{M}$ cPTIO(INO), whose EPR spectrum is also shown in Figure 1. In line with previous literature, cPTIO(NNO) gives a five-line EPR spectrum, characterized by hyperfine splitting due to the presence of two equivalent $\mathrm{N}$ nuclei, while cPTIO(INO) shows the specific seven-peak spectrum due to the presence of two non-equivalent $\mathrm{N}$ nuclei.

To assess the spin trap stability in the presence of biological samples, a series of experiments were performed in vivo on Arabidopsis cultured cells, by incubating 5-day-old cell cultures with $100 \mu \mathrm{M}$ cPTIO(NNO) or cPTIO(INO). The EPR measurements were done on the culture medium after different incubation times (from 1 to $130 \mathrm{~min}$ ). It was observed that the intensity of EPR signals of both cPTIO(NNO) and cPTIO(INO) rapidly decreased in the first minutes of incubation, reaching nearly zero after $130 \mathrm{~min}$ (Figure 2). The disappearance of $\mathrm{cPTIO}(\mathrm{NNO})$ signal was not followed by the appearance of $\mathrm{CPTIO}(\mathrm{INO})$ spectrum.

In order to verify whether the reduction of CPTIO EPR signal was associated with the presence of a cell-linked activity, EPR measurements were performed incubating cPTIO(NNO) either in exhausted culture medium, withdrawn from 5-day-old cell cultures, or in the presence of boiled 5-day-old cell cultures. In both cases, the intensity of EPR signals was maintained for longer time compared with the previous experiments, with a signal decrease of less than $10 \%$ after $180 \mathrm{~min}$ (Figure 3).

Two hypotheses can explain why the intensity of EPR signals rapidly decreases in cell cultures. The first is a fast uptake of cPTIO, which accumulates inside the cells, so that it becomes not measurable in the culture medium; the second is that CPTIO is rapidly transformed in an EPR silent product, either inside, after uptake, or outside the cells.

To clarify this point, $100 \mu \mathrm{M}$ cPTIO was incubated with Arabidopsis cell cultures for $10 \mathrm{~min}$. After this time, the cells were separated from the medium and the EPR signal was measured both in the medium and in the total soluble cell extract. The intensity of the EPR signal measured in the external medium significantly decreased after $10 \mathrm{~min}$ of incubation (Figure 4). A
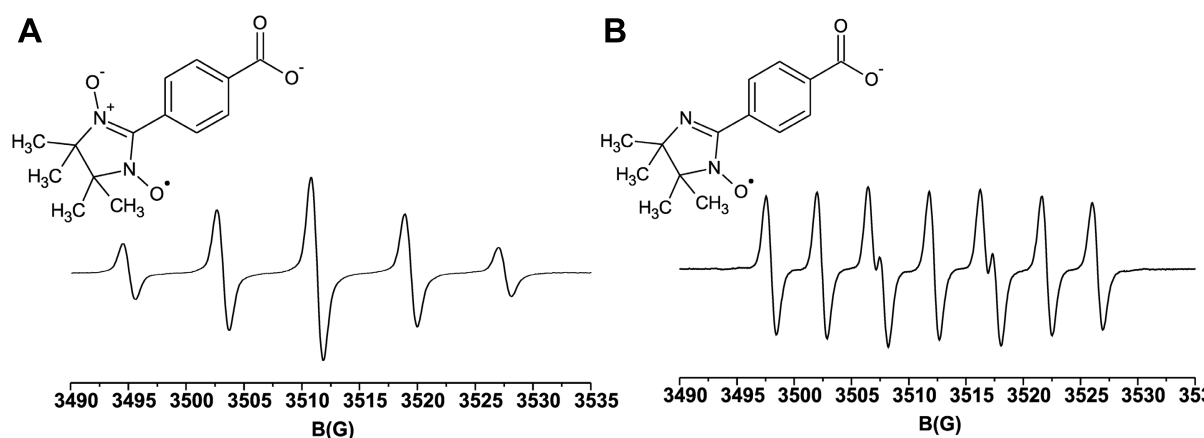

$\begin{array}{llllllllll}3490 & 3495 & 3500 & 3505 & 3510 & 3515 & 3520 & 3525 & 3530 & 3535\end{array}$ B(G)

FIGURE 1 | Molecular structures and room temperature EPR spectra. (A) $100 \mu \mathrm{M}$ cPTIO(NNO); (B) $100 \mu \mathrm{M}$ cPTIO(INO), in water. 


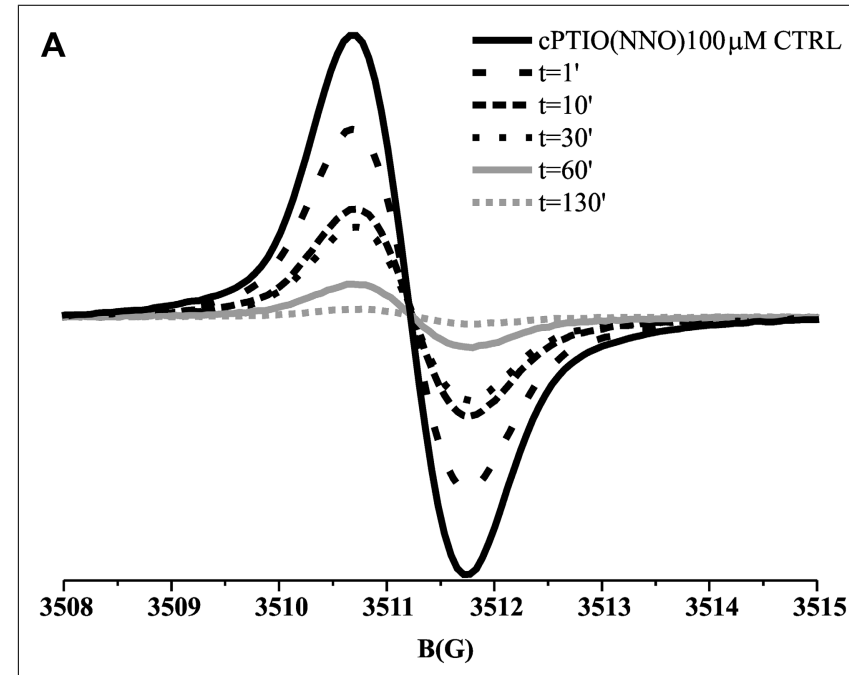

B

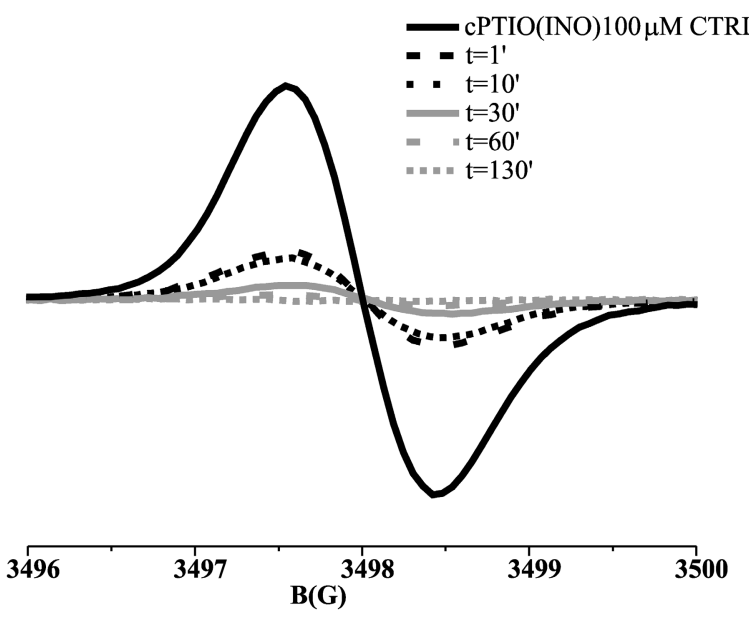

C

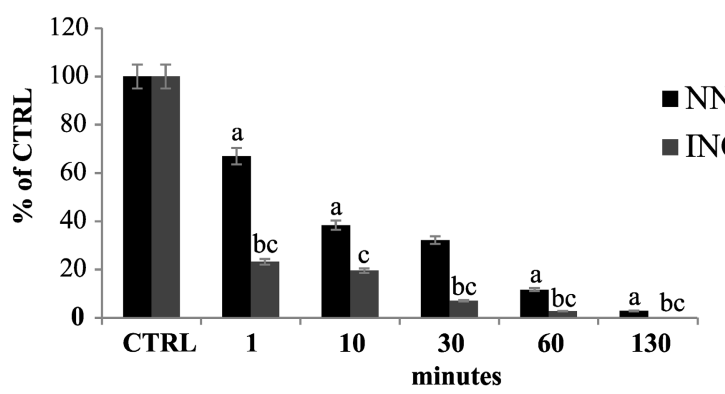

FIGURE 2 (Time dependence of cPTIO(NNO) and cPTIO(INO) EPR signals in suspension cultured cells. Central line of $c P T I O(N N O)$ (A) and low field, first line of cPTIO(INO) (B) EPR spectra were quantified. In (C) the intensity of EPR signal for each measurement, is presented as percentage of the total signal resulting from measurement of $100 \mu \mathrm{M}$ cPTIO(NNO) or (INO) dissolved in water (CTRL) \pm SD. cPTIO(NNO) or (INO) was added to the supernatant of 5-day-old Arabidopsis suspension cultured cells and aliquots of the medium were collected at the time points indicated. A Student's $t$-test was performed for each experiment and statistically significant data are marked: (a) $p<0.01 \mathrm{cPTIO}$ (NNO) compared with the previous time point, (b) $p<0.01 \mathrm{cPTIO}$ (INO) compared with the previous time point, (c) $p<0.01 \mathrm{cPTIO}$ (INO) compared with $\mathrm{CPTIO}$ (NNO) at the same time point.

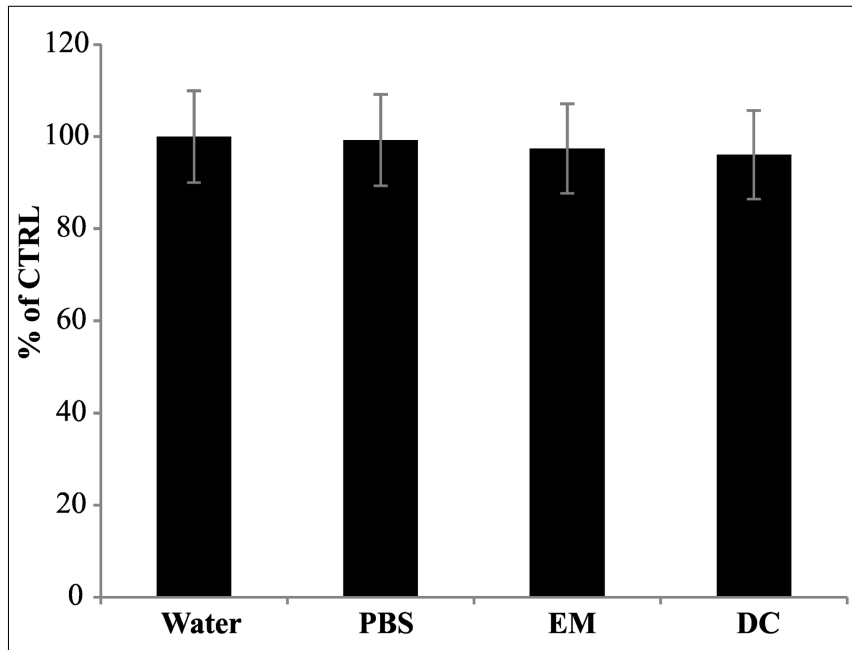

FIGURE 3 | Electron paramagnetic resonance signal of cPTIO incubated in water, PBS, exhausted medium or with dead cells. CPTIO(NNO) was incubated in water, PBS, exhausted medium (EM) or with boiled dead cells (DC). EPR spectra of the samples were detected after 180 min of incubation. Intensities of EPR signals are given as percentage of the total signal at $t_{0}$.

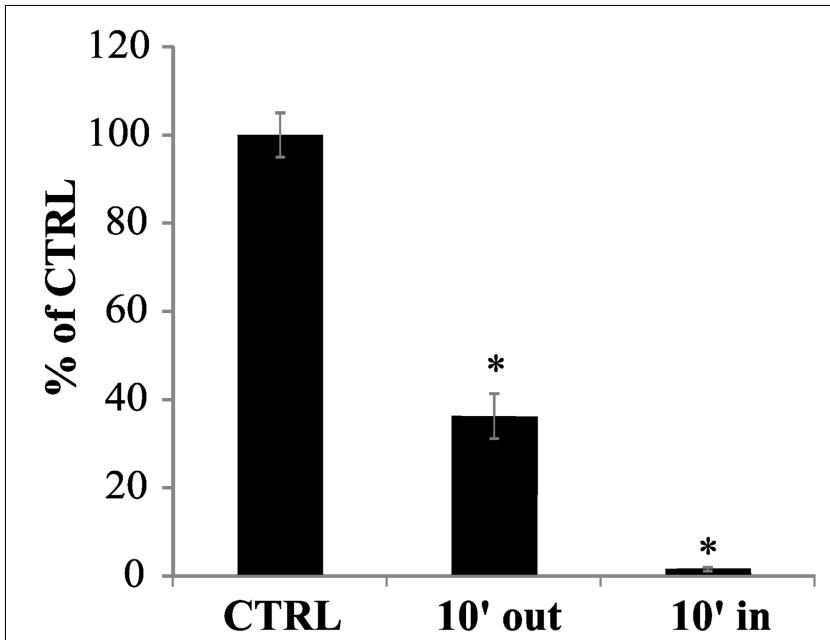

FIGURE 4 | cPTIO uptake in cell cultures. cPTIO(NNO) was incubated with cell cultures. At 10 min a sample from the culture medium was collected (10' out) and all cells were harvested. The cells were disrupted and centrifuged. An aliquot of the supernatant was collected (10' in). EPR signals were detected and given as percentage of the total signal resulting from measurement of $100 \mu \mathrm{M}$ cPTIO(NNO) dissolved in water

$(C T R L) \pm S D$. A Student's $t$-test was performed and samples statistically different from the CTRL marked with an asterisk

small cPTIO(NNO) signal was detected also in the cell extract, showing that cPTIO was actually entering the cells but its concentration resulted strongly reduced when compared to the bulk concentration initially added to the sample (about $1 \%$ of the signal of $100 \mu \mathrm{M}$ cPTIO in water; Figure 4). This result proves that the decrease of the EPR signal observed in the medium is not due to the spin trap accumulation inside the cells, but rather to the disappearance of cPTIO. 
The degradation of cPTIO(INO) by the cells, at a faster rate compared to that of $\mathrm{CPTIO}(\mathrm{NNO})$ (Figure 2), implies that, for an in vivo quantitative measurement of $\mathrm{NO}$ via $\mathrm{EPR}$, the use of cPTIO is not feasible because cPTIO(INO) is not stable and does not accumulate in a steady state concentration reaching the sensitivity of the EPR technique. On the other hand, the fact that $100 \mu \mathrm{M}$ cPTIO(NNO) disappears in a short time (with a decay time constant of about $15 \mathrm{~min}^{1}$ ) and in a measurable way indicates that the endogenous $\mathrm{NO}$, present in low concentration, is not the main responsible for the reactions undergone by $\mathrm{CPTIO}(\mathrm{NNO})$. This hypothesis was also supported by a series of experiments on cultured cells treated with salicylic acid (SA), which induces an increase of NO production (Zottini et al., 2007), to evaluate influence of $\mathrm{NO}$ on the decay rate of cPTIO(NNO) EPR signal. In that instance, it was found that the decay rate of cPTIO(NNO) was not affected by the treatment (not shown) meaning that the main reason for $\mathrm{CPTIO}(\mathrm{NNO})$ disappearance was not the reaction with $\mathrm{NO}$ but with other substrates such as reductans present in the cells (Haseloff et al., 1997). Thus, both the fast transformation of cPTIO(INO) and the competitive reactions of cPTIO(NNO) with substrates others than NO contribute to hinder the quantification of NO in living cells by using cPTIO as spin trap.

\section{cPTIO NO SCAVENGING EFFICACY IN IN VIVO MEASUREMENTS}

cPTIO is widely used as NO scavenger in plant experimental systems to validate the involvement of $\mathrm{NO}$ in pathways triggered by different external/internal stimuli. cPTIO is used in plant cell cultures but also in experiments carried out on plant seedlings. Therefore, the kinetics of cPTIO reactions was also examined in this experimental system. The experiments were performed on Arabidopsis 8-day-old seedlings, incubated in $50 \mathrm{ml}$ of liquid medium, by adding $100 \mu \mathrm{McPTIO}(\mathrm{NNO})$ to the external medium. The EPR measurements were performed on the culture medium after several incubation times (from 1 to $130 \mathrm{~min}$ ). As shown in Figure 5, a decrease of EPR signal associated to CPTIO was observed, but it was slower when compared to that of cell cultures. A possible explanation for different decreasing rates could be the much more complex and slower process of cPTIO uptake in the whole plant compared to cultured cells. Thus, the uptake may become a rate-determining step in the cPTIO EPR signal disappearance. The decrease in the EPR signal of cPTIO was not accompanied by the formation of the INO EPR signal in cell cultures, as well as in seedlings.

The experiments performed on boiled cell culture reported above indicated that the disappearance of the cPTIO EPR signal was dependent on a cell-linked activity. To validate this hypothesis the stability of cPTIO incubated with different amount of Arabidopsis total soluble extract was investigated. Figure 6 shows the time dependence of the $100 \mu \mathrm{M} c \mathrm{PTIO}(\mathrm{NNO})$ and (INO) EPR signals following the addition of different amounts of extract. The intensity of EPR signals strongly decreased depending on both the incubation time and the extract concentration. This result strongly supports an enzyme-dependent transformation of the chemical compounds. cPTIO(INO) showed a faster decay rate compared to cPTIO(NNO), using the same

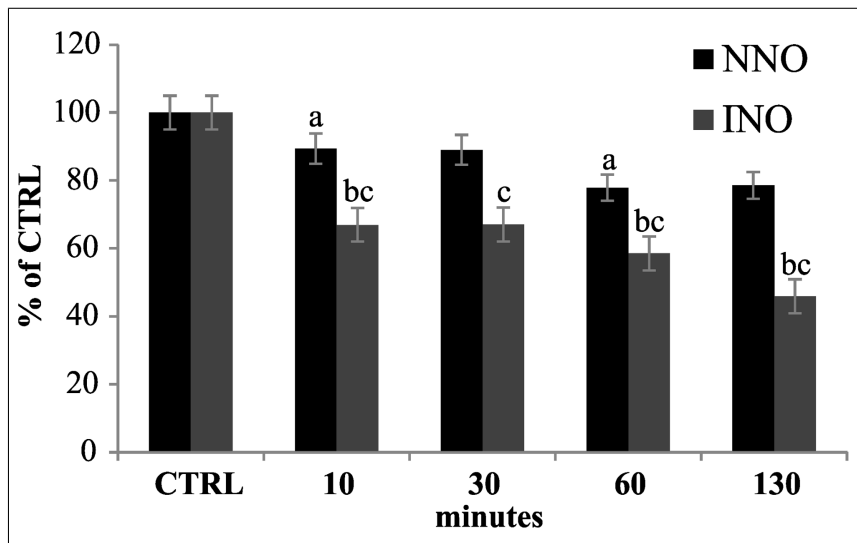

FIGURE 5 | Time dependence of cPTIO EPR signals in seedlings. 8-day-old Arabidopsis seedlings were incubated in $50 \mathrm{ml}$ of liquid culture medium. CPTIO(NNO) or (INO) was added to the supernatant and aliquots of the medium were taken at indicated time points. EPR measurements are presented as percentage of the total signal resulting from measurement of $100 \mu \mathrm{M}$ cPTIO(NNO) and (INO) dissolved in water (CTRL) \pm SD. A Student's t-test was performed for each experiment and statistically significant data marked: (a) $p<0.01 \mathrm{cPTIO}$ (NNO) compared with the previous time point, (b) $p<0.01 \mathrm{cPTIO}$ (INO) compared with the previous time point, (c) $p<0.01 \mathrm{cPTIO}$ (INO) compared with cPTIO(NNO) at the same time point

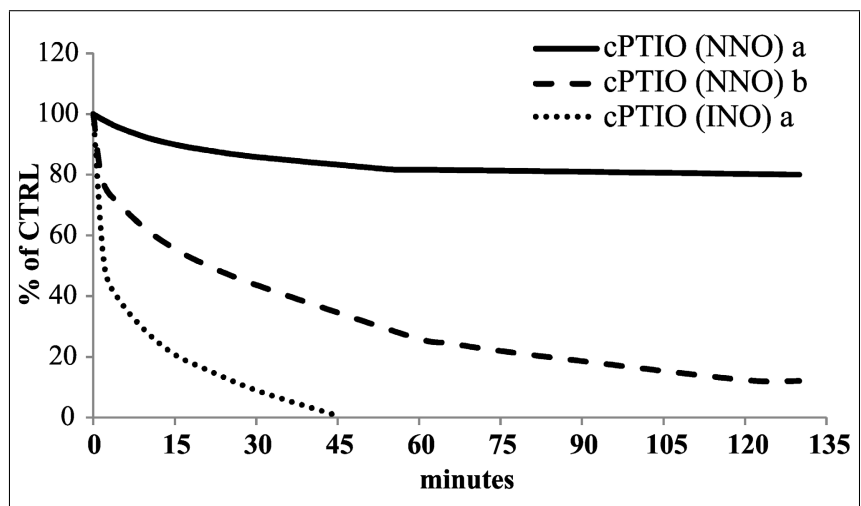

FIGURE 6 | Time dependence of cPTIO EPR signals in Arabidopsis total soluble extract. $100 \mu \mathrm{M}$ cPTIO(NNO) or (INO) was incubated in $50 \mathrm{mM}$ PBS pH 7 in the presence of a protein concentration of (a) $0.3 \mathrm{mg} / \mathrm{ml}$ or (b) $1.8 \mathrm{mg} / \mathrm{ml}$ Arabidopsis total extract. The time course of the reactions was followed as decrease of the EPR signals. The EPR signals for each measurement were presented as percentage of the total signal resulting from measurement of $100 \mu \mathrm{M}$ cPTIO dissolved in PBS. The plot reported is representative of three independent experiments.

concentration of total extract. Moreover the EPR signal of cPTIO(INO) in the presence of the higher concentration of total soluble extract $(1.8 \mathrm{mg} / \mathrm{ml})$ was not even detectable (data not shown).

A common method for NO detection is the use of DAF-FM fluorescent dye and its membrane-permeable diacetate form (Kojima et al., 1998). DAF-FM has been used to localize NO production site in plant cells and tissues (Correa-Aragunde et al., 2004), and quantify the production of NO in suspension cultured cells (Krause and Durner, 2004). In each of these studies, fluorescence quenching by cPTIO has been used as confirmation that DAF-FM fluorescence was indeed due to NO (Gupta and Igamberdiev, 2013), 


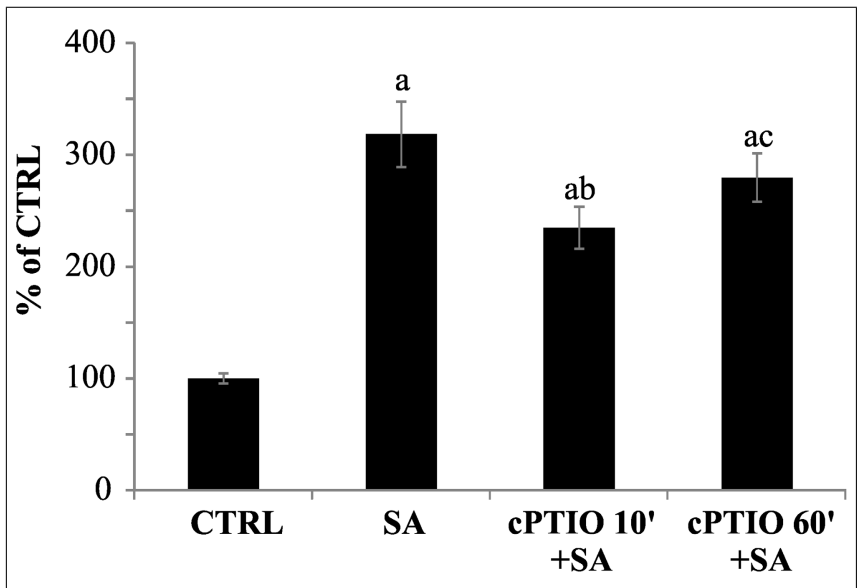

FIGURE 7 | Time dependence of DAF-FM fluorescence quenching by cPTIO. $100 \mu \mathrm{M}$ cPTIO(NNO) was added to cell cultures and kept for different incubation times (10 or $60 \mathrm{~min}$ ) before $1 \mathrm{mM}$ salicylic acid (SA) addition. NO levels were analyzed 60 min after SA treatment by using DAF-FM (excitation: $488 \mathrm{~nm}$, detection: $515 \mathrm{~nm}$ ). Signal from 3D reconstruction was quantified by image densitometry and reported as percentage of the not treated sample $(C T R L) \pm S D$. A Student's $t$-test was performed for each experiment and statistically significant data marked: (a) $p<0.01$ compared with CTRL, (b) $p<0.01$ compared with SA, (c) $p<0.01$ compared with CPTIO $10^{\prime}+\mathrm{SA}$.

since cPTIO is known to be acting as a specific NO scavenger. However, it has been shown that in the presence of high levels of NO, cPTIO can induce an increase of DAF-FM fluorescence, rather than a quenching, through a complex pathway of oxidation reactions (Vitecek et al., 2008).

The experimental data reported here have proven that cPTIO is rapidly transformed in an EPR silent compound in samples containing cells or seedlings. Thus, it is important to understand whether the reaction products of CPTIO are still able to scavenge NO. To evaluate this, cell cultures stimulated by SA were pretreated for different incubation times with CPTIO, and NO was detected by DAF-FM. In a previous paper (Zottini et al., 2007), NO production induced by SA in Arabidopsis cell cultures has been already reported and in that case it was measured with DAF-FM and oxyhemoglobin, in parallel. The two techniques showed indeed comparable results, confirming NO production triggered by SA.

As reported in Figure 7, the increasing of cPTIO incubation time leads to a reduction of its scavenging efficacy. While cPTIO pre-incubated for $10 \mathrm{~min}$ is able to scavenge SA-induced NO, a longer pre-incubation significantly decreases the scavenging efficacy. These results demonstrate that molecules deriving from cPTIO cell reactions are not able to scavenge NO.

\section{DISCUSSION}

In the present work, we provide a systematic study to evaluate the efficacy of using cPTIO as NO spin trap and NO scavenger in plant systems, in particular, in cell cultures and seedlings.

The nitronyl nitroxides have been already used in vitro and in animal systems as spin traps for NO (Woldman et al., 1994; Haseloff et al., 1997) because of their specificity for NO compared to other spin traps, such as iron dithiocarbamates or oxyhemoglobin (Hogg, 2010).
It has been reported that in those systems cPTIO(NNO) and (INO) are transformed in the EPR silent form hydroxylamine (Woldman et al., 1994; Haseloff et al., 1997). The occurring reaction is likely a reduction associated to the presence of reducing substrates, such as glutathione and/or ascorbate, in the cell environment. Superoxide has also been reported as a possible reductant of nitroxides (Haseloff et al., 1997).

Our results strongly suggest that, also in plant cells, different reducing species may react with cPTIO. Therefore, the use of nitronyl nitroxides as spin traps for $\mathrm{NO}$ detection via EPR in plant systems, where endogenous rates of $\mathrm{NO}$ generation are very low, is compromised by their very rapid reduction into diamagnetic EPR silent products.

We also evidenced that the reduction of cPTIO is an enzymemediated process. In fact, it was observed that $\mathrm{CPTIO}(\mathrm{NNO})$ and cPTIO(INO) EPR signals did not decrease in fresh culture medium (data not shown), in exhausted medium, or in boiled cell suspensions. As expected, their decay rates increased after the addition of cellular extract.

Summarizing, the competitive reactions of cPTIO, and the fast reduction of $\mathrm{CPTIO}$ (INO), make the use of $\mathrm{CPTIO}$ as spin trap for NO detection via EPR unmanageable, at least in the micromolar range of NO concentrations.

The other question addressed was whether the use of cPTIO as NO scavenger was reliable, in spite of all the occurring transformation events. To shed light on this controversial point, we carried out a series of experiments with plant cells and seedlings. The results clearly indicate that also the scavenging abilities of cPTIO may be impaired due to cellular reactions. Actually, we observed that when NO production was induced by SA, the scavenging efficacy of $100 \mu \mathrm{M}$ cPTIO was significantly reduced in a time-dependent manner. We, thus, infer that to obtain a strong scavenging effect, a higher concentration of $\mathrm{CPTIO}$ should be used. On the other hand, it has to be kept into consideration that high concentrations of cPTIO can give rise to artifacts, when DAF-FM is used as detection method (Arita et al., 2006).

In conclusion, the reported analysis underlines the drawbacks of using cPTIO as EPR probe for in vivo measurements of NO in plants. In addition, the results provide helpful indication for the right use of cPTIO as NO scavenger. In fact, in order to effectively scavenge NO, the parallel depletion of cPTIO in living cells has to be taken into account. The relatively low cPTIO concentration used in our experiments has allowed us to evidence better the time dependence of $\mathrm{cPTIO}$ degradation, confirming data obtained by EPR measurements. At the same time, it is evidenced that the use of low concentration of cPTIO could compromise its scavenging efficacy due to competitive reactions.

The complex chemical behavior of cPTIO in plant environment may explain why cPTIO is not always able to completely scavenge $\mathrm{NO}$, especially for treatments inducing a gradual and continuous production of NO.

Since CPTIO is highly specific to NO, its use remains valuable. However, to produce significant data, and observe the scavenging effect of cPTIO, concentrations and incubation time should be accurately chosen, depending on the analyzed system and in relation to the amount of NO produced. 


\section{MATERIAL AND METHODS CHEMICALS}

2-4-Carboxyphenyl-4,4,5,5-tetramethylimidazoline-1-oxyl-3oxide (Alexis Biochemicals ALX-430-001), DAF-FM-DA (Alexis Biochemicals, ALX-620-071), SA (S7401 SIGMA), MS medium salt including vitamins (Duchefa M 0409), MAHMA NONOate (Alexis, Vinci, Italy).

\section{CELL CULTURES}

Suspension cell culture was generated from hypocotyls dissected from young plantlets of Arabidopsis (ecotype Landsberg erecta) and subcultured in AT3 medium (Desikan et al., 1996). For subculture cycles, $5 \mathrm{ml}$ of cell culture volume [0.8 g fresh weight (FW)] was placed in $100 \mathrm{ml}$ Erlenmeyer flasks containing $45 \mathrm{ml}$ of liquid medium. Cells were subcultured in fresh medium at 7 days intervals and maintained in a climate chamber on a horizontal rotary shaker $(80 \mathrm{rpm})$ at $24^{\circ} \mathrm{C}$ with a $16-/ 8$-h photoperiod and a light intensity of $70 \mathrm{mmol} \mathrm{m} \mathrm{m}^{-2} \mathrm{~s}^{-1}$. All analyses and treatments with filter-sterilized solutions of SA were carried out with 5-day-old cultures $(4 \mathrm{~g} \mathrm{FW})$.

\section{Arabidopsis SEEDLINGS}

Seeds of Arabidopsis (ecotype Columbia) were surface sterilized by washing with $70 \% \mathrm{EtOH}, 0.05 \%$ Triton X 100. After the sterilization they were grown on MS - $1 / 2$ medium supplemented with $0.5 \mathrm{~g} / \mathrm{l} \mathrm{MES}-\mathrm{KOH}$ pH 5.7, 0.8\% plant agar, and 1\% sucrose. After $48 \mathrm{~h}$ of incubation at $4^{\circ} \mathrm{C}$ in the dark, plates were put in a growing chamber at $22^{\circ} \mathrm{C}$ and long day light period ( $16 \mathrm{~h} \mathrm{light/} 8 \mathrm{~h}$ dark). The plates were kept vertically. Seedlings of 8 days were used for the experiments ( $4 \mathrm{~g} \mathrm{FW})$.

\section{Arabidopsis TOTAL SOLUBLE EXTRACT}

$100 \mathrm{mg}$ of Arabidopsis cells or seedlings were homogenized by Eppendorf micropestle in extraction buffer added 1:1 w/v $(50 \mathrm{mM}$ PBS pH 7, EDTA $1 \mathrm{mM}$, protease inhibitor cocktail). The samples were centrifuged $1 \mathrm{~min}$ at $16000 \times g$ at $4^{\circ} \mathrm{C}$. The supernatant was recovered and quantified by Bradford protein assay test (Biorad). A protein content of 0.3 or $1.8 \mathrm{mg} / \mathrm{ml}$ was used in each experiment.

\section{cPTIO ANALYSES}

$100 \mu \mathrm{M}$ cPTIO(NNO) or cPTIO(INO) was added directly to the Arabidopsis cells culture. Aliquots of the medium were collected at different incubation time, and immediately frozen in liquid nitrogen. Samples were then analyzed by EPR spectroscopy at room temperature, after thawing.

The experiments with boiled dead cells were performed using Arabidopsis cell cultures, boiled for $30 \mathrm{~min}$.

The experiments with exhausted medium were performed incubating $100 \mu \mathrm{M}$ cPTIO(NNO) in the medium withdrawn from 5-day-old cell cultures.

\section{REFERENCES}

Arita, N. O., Cohen, M. F., Tokuda, G., and Yamasaki, H. (2006). Fluorometric detection of nitric oxide with diaminofluoresceins (DAFs): applications and limitations for plant NO research. Plant Cell Monogr. 5, 269280. doi: 10.1007/7089_2006_097
Berliner, L. J. (2000). In Vivo EPR(ESR): Theory and Applications (Biological Magnetic Resonance). New York: Kluwer Academic/Plenum Publishing Corp.

Correa-Aragunde, N., Graziano, M., and Lamattina, L. (2004). Nitric oxide plays a central role

8 days-old Arabidopsis seedlings (4 g FW) were incubated in $50 \mathrm{ml}$ liquid culture medium (MS - 1/2 medium supplemented with $0.5 \mathrm{~g} / \mathrm{l} \mathrm{MES}-\mathrm{KOH} \mathrm{pH} 5.7,0.8 \%$ plant agar, and 1\% sucrose). $100 \mu \mathrm{M}$ cPTIO was added to the medium. Aliquots of the medium were analyzed by EPR.

$100 \mu \mathrm{M}$ cPTIO was incubated with 0.3 or $1.8 \mathrm{mg} / \mathrm{ml}$ total soluble extract concentration, diluted in PBS and added to the capillary for EPR measurements.

The EPR signals for each measurement were presented as percentage of the total signal resulting from measurement of $100 \mu \mathrm{M}$ cPTIO dissolved in water.

\section{STATISTICAL ANALYSES}

All experiments were performed at least three times on independent biological replicates. The results are presented as mean $\pm \mathrm{SD}$ (standard deviation). Statistical differences were determined by using Student's $t$-test. Statistical significance was assigned at $p<0.01$.

\section{DAF-FM ANALYSES}

2-4-Carboxyphenyl-4,4,5,5-tetramethylimidazoline-1-oxyl-3oxide was added to different flasks of Arabidopsis cell culture of 5 days $(4 \mathrm{~g} \mathrm{FW})$ and incubated for different pre-incubation times. After the cPTIO pre-incubation $15 \mu \mathrm{M}$ DAF-FM-DA was loaded in the cells as previously described (Zottini et al., 2007). $1 \mathrm{mM}$ SA was added to the cell culture and cells were analyzed after $60 \mathrm{~min}$ of treatment. Samples were observed by confocal microscopy using the 488 Argon line for excitation. 3D reconstruction of the cells were obtained by Nikon PCM2000 (Biorad) laser scanning confocal microscope. DAF-FM Fluorescence was quantified by image densitometry analysis of the pixel intensities using Image software (NIH, USA). At least 20 cells per samples were singularly analyzed.

\section{EPR SPECTROSCOPY}

Room temperature continuous wave EPR spectra were collected using a Bruker Elexsys E580-X-band spectrometer equipped with the Elexsys Super High Sensitivity Probehead. All measurements were performed in capillaries (ID $0.9 \mathrm{~mm} ; 50 \mu \mathrm{l}$ total volume). Acquisition parameters were the following: microwave frequency $=9.86 \mathrm{GHz}$; modulation amplitude in the range $0.15-$ 0.3 Gauss, microwave power $=6.370 \mathrm{~mW}$; sweep time $167.77 \mathrm{~s}$, time constant $40.96 \mathrm{~ms}$.

\section{ACKNOWLEDGMENTS}

We would like to acknowledge the financial support of University of Padova (PRAT 2009-CPDA093805/09) to Donatella Carbonera and Michela Zottini.

in determining lateral root development in tomato. Planta 218, 900-905. doi: 10.1007/s00425-0031172-7

Desikan, R., Hancock, J. T., Coffey, M. J., and Neill, S. J. (1996). Generation of active oxygen in elicited cells of Arabidopsis thaliana is mediated by a NADPH oxidase-like enzyme. FEBS Lett. 382, 213-217. doi: 10.1016/0014-5793(96)00177-9

Gupta, K. J., Fernie, A. R., Kaiser, W. M., and van Dongen, J. T. (2010). On the origins of nitric oxide. Trends Plant Sci. 16, 160-168. doi: 10.1016/j.tplants.2010.11.007 
Gupta, K. J., and Igamberdiev, A. U. (2013). Recommendations of using at least two different methods for measuring NO. Front. Plant. Sci. 4:58. doi: 10.3389/fpls.2013. 00058

Haseloff, R. F., Zöllner, S., Kirilylik, I. A., Grigorev, I. A., Reszka, R., Bernhardt, R., et al. (1997). Superoxide-mediated reduction of the nitroxide group can prevent detection of nitric oxide by nitronyl nitroxides. Free Radic. Res. 26, 7-17. doi: 10.3109/10715769 709097780

Hogg, N. (2010). Detection of nitric oxide by electron paramagnetic resonance spectroscopy. Free Radic. Biol. Med. 49, 122-129. doi: 10.1016/j.free radbiomed.2010.03.009

Hogg, N., Singh, R. J., Joseph, J., Neese, F., and Kalyanaraman, B. (1995). Reactions of nitric oxide with nitronyl nitroxides and oxygen: prediction of nitrite and nitrate formation by kinetic simulation. Free Radic. Res. 22, 47-56. doi: 10.3109/ 10715769509147527

Kojima, H., Nakatsubo, N., Kikuchi, K., Urano, Y., Higuchi, T., Tanaka, J., et al. (1998). Direct evidence of NO production in rat hippocampus and cortex using a new fluorescent indicator: DAF2 DA. Neuroreport 9, 3345-3348. doi: 10.1097/00001756-19981026000001

Kramer, E. M., and Bennett, M. J. (2006). Auxin transport: a field in flux. Trends Plant Sci. 11,
382-386. doi: 10.1016/j.tplants.2006. 06.002

Krause, M., and Durner, J. (2004). Harpin inactivates mitochondria in Arabidopsis suspension cells. Mol. Plant Microbe Interact. 17, 131139. doi: 10.1094/MPMI.2004.17. 2.131

Mur, L. A., Mandon, J., Persijn, S., Cristescu, S. M., Moshkov, I. E., Novikova, G. V., et al. (2013). Nitric oxide in plants: an assessment of the current state of knowledge. $A o B$ Plants 5, pls052. doi: 10.1093/aob$\mathrm{pla} / \mathrm{pls} 052$

Rumer, S., Krischke, M., Fekete, A., Mueller, M. J., and Kaiser, W. M. (2012). DAF-fluorescence without NO: elicitor treated tobacco cells produce fluorescing DAFderivatives not related to DAF-2 triazol. Nitric Oxide 27, 123135. doi: 10.1016/j.niox.2012. 05.007

Terrile, M. C., Paris, R., CalderonVillalobos, L. I., Iglesias, M. J., Lamattina, L., Estelle, M., et al. (2012). Nitric oxide influences auxin signaling through $S$-nitrosylation of the Arabidopsis TRANSPORT INHIBITOR RESPONSE 1 auxin receptor. Plant J. 70, 492-500. doi: $\quad 10.1111 /$ j.1365-313X.2011.04 885. $\mathrm{x}$

Vanin, A. F., Liu, X., Samouilov, A., Stukan, R. A., and Zweier, J. L. (2000). Redox properties of irondithiocarbamates and their nitrosyl derivatives: implications for their use as traps of nitric oxide in biological systems. Biochim. Biophys. Acta 1474, 365-377. doi: 10.1016/S0304-4165 (00)00033-7

Vitecek, J., Reinohl, V., and Jones, R. L. (2008). Measuring NO production by plant tissues and suspension cultured cells. Mol. Plant 1, 270-284. doi: 10.1093/mp/ ssm020

Wang, Y., Lin, A., Loake, G. J., and Chu, C. (2013). $\mathrm{H}_{2} \mathrm{O}_{2}$-induced leaf cell death and the crosstalk of reactive nitric/oxygen species. J. Integr. Plant Biol. 55, 202-208. doi: 10.1111/ jipb.12032

Woldman, Y. Y., Khramtsov, V. V., Grigorev, I. A., Kiriljuk, I. A., and Utepbergenov, D. I. (1994). Spin trapping of nitric oxide by nitronylnitroxides: measurement of the activity of no synthase from rat cerebellum. Biochem. Biophys. Res. Commun. 202, 195-203. doi: 10.1006/bbrc. 1994 1912

Ye, W., Hossain, M. A., Munemasa, S., Nakamura, Y., Mori, I. C., and Murata, Y. (2013). Endogenous abscisic acid is involved in methyl jasmonate-induced reactive oxygen species and nitric oxide production but not in cytosolic alkalization in Arabidopsis guard cells. J. Plant Physiol. 170, 1212 1215. doi: 10.1016/j.jplph.2013. 03.011

Yoshioka, T., Iwamoto, N., and Ito, K. (1996). An application of electron paramagnetic resonance to evaluate nitric oxide and its quenchers. J. Am. Soc. Nephrol. 7, 961-965.
Zottini, M., Costa, A., De Michele, R. Ruzzene, M., Carimi, F., and Lo Schiavo, F. (2007). Salicylic acid activates nitric oxide synthesis in Arabidopsis. J. Exp. Bot. 58, 1397-1405. doi: 10.1093/jxb/erm001

Conflict of Interest Statement: The authors declare that the research was conducted in the absence of any commercial or financial relationships that could be construed as a potential conflict of interest.

Received: 27 May 2013; accepted: 13 August 2013; published online: 29 August 2013.

Citation: D'Alessandro S, Posocco B, Costa A, Zahariou G, Lo Schiavo F, Carbonera D and Zottini M (2013) Limits in the use of cPTIO as nitric oxide scavenger and EPR probe in plant cells and seedlings. Front. Plant Sci. 4:340. doi: 10.3389/fpls.2013.00340

This article was submitted in Plant Physiology, a section of the journal Frontiers in Plant Science.

Copyright (c) 2013 D'Alessandro, Posocco, Costa, Zahariou, Lo Schiavo, Carbonera and Zottini. This is an open-access article distributed under the terms of the Creative Commons Attribution License (CC BY). The use, distribution or reproduction in other forums is permitted, provided the original author(s) or licensor are credited and that the original publication in this journal is cited, in accordance with accepted academic practice. No use, distribution or reproduction is permitted which does not comply with these terms. 